PFLEGE-IMMOBILIEN

\title{
Licht und Schatten
}

Rainer Jurowsky

In den vergangenen Jahren hat sich bei den Immobilieninvestitionen privater Kapitalanleger ein uneinheitliches Bild gezeigt. Die in Aussicht gestellten Renditeerwartungen sind-aus unterschiedlichen Gründen - oftmals nicht realisiert worden.

Wenn sich auch mit dem derzeit zu beobachtenden Wirtschaftsaufschwung ebenfalls die Aussichten auf dem Immobilienmarkt aufhellen, suchen Investoren dennoch vor dem Hintergrund der Erfahrungen aus der Vergangenheit nach Investitionsalternativen. Besonders gefragt sind hierbei Immobilien, die (vermutlich) in der Zukunft vermehrt nachgefragt werden, weil der entsprechende Bedarf besteht.

Der Blick fällt hierbei vorrangig auf Spezialimmobilien, die ein spezielles Nachfragesegment bedienen. Angesichts der demografischen Entwicklung, die Deutschland in den nächsten Jahrzehnten bevorsteht, verwundert es nicht, dass die Immobilienwirtschaft auch die »Seniorenimmobilie « - und hier insbesondere die Pflegeimmobilie - als Investitionsobjekt entdeckt hat. Das private Kapital, das zweifellos auf dem Markt vorhanden ist, soll nun in diesen Markt fließen, weil die hierdurch finanzierten Immobilien in Zukunft stark nachgefragt werden. Vermutet werden hohe Auslastungsquoten und dadurch sowohl attraktive Renditen als auch ein geringes Risiko für private Investoren.

\section{Demografie}

Aufgrund der demografischen Entwicklung wird der Anteil der alten Menschen an der Gesamtbevölkerung größer werden. Nach den jüngsten Prognosen des Statistischen Bundesamtes wird die Anzahl der Menschen, die älter als 65 Jahre sind, von derzeit 13,3 Millionen auf 21,6 Millionen im Jahre 2030 steigen. Gleichzeitig wird bis 2030 auch die Anzahl der über 80-Jährigen von heute 3,25 Millionen auf sechs Millionen ansteigen. Hierbei handelt es sich nicht um mögliche Szenarien, bei denen noch gegengesteuert werden kann. Die Bevölkerungsentwicklung ist mit einem Tanker zu vergleichen, der auch nicht innerhalb kurzer Zeit gestoppt werden kann.

Gleichzeitig zu der Zunahme der Bevölkerungsgruppe der über 80-Jährigen wird auch die Anzahl der pflegebedürftigen Menschen zunehmen, denn das Risiko der Pflegebedürftigkeit ist unmittelbar an das zunehmende Lebensalter gekoppelt. In dieser Bevölkerungsgruppe ist zudem das Problem der Mehrfacherkrankungen, verbunden mit gerontopsychiatrischen Veränderungen, stark

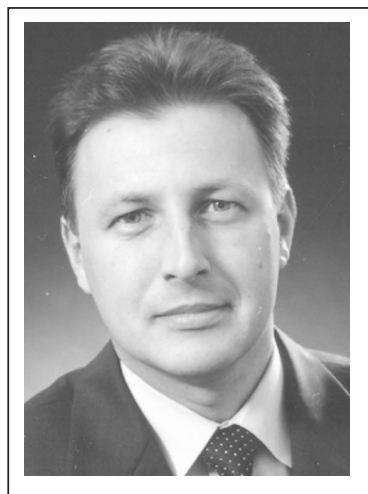

Dr. Rainer Jurowsky, Wirtschaftsprüfer und Steuerberater, ist Professor für Allgemeine Betriebswirtschaftslehre, Betriebliche Steuerlehre und Unternehmensprüfung an der Fachhochschule Düsseldorf. Er ist Gründer der »agecare consult $\mathrm{GmbH}$ «(Bonn), die sich mit der Entwicklung von

Pflege- und Betreuungskonzepten für ältere Menschen sowie der Immobilienentwicklung in diesem Bereich beschäftigt.

E-Mail rainer.jurowsky@fh-duesseldorf.de

verbreitet. Der medizinische Fortschritt wird lediglich dazu führen, dass die Gesamtlebenserwartung der Menschen - unter Umständen trotz lebensbedrohlicher Krankheiten - weiter ansteigt. Eine generelle Verbesserung des Gesundheitszustands dieser Bevölkerungsschichten wird nicht erreicht werden. Da insbesondere die Präventivmedizin in den vergangenen Jahren vernachlässigt wurde, werden die Menschen in Deutschland zwar immer älter, nicht aber unbedingt gesünder.

Der Jahrgang 1964 war der bislang geburtenreichste Jahrgang in Deutschland. Dieser Jahrgang ist heute 42 Jahre alt. In 30 bis 40 Jahren wird sich für diesen Jahrgang das Problem der Pflegebedürftigkeit mit hoher Dringlichkeit stellen.

\section{Renditekriterien}

Angesichts der dargestellten demografischen Entwicklung in Deutschland und des gleichzeitig bestehenden Kapitalangebotes privater Investoren scheint eine Investition privater Kapitalgeber in diesem Bereich lohnend zu sein. Zwar ist der Markt derzeit sehr intransparent und zersplittert - so werden die über 9.000 stationären Pflegeeinrichtungen in Deutschland von rund 6.500 Betreibern unterhalten - und in der Vergangenheit sind einige private Investitionen in diesem Bereich gescheitert, aber dennoch stehen wir vor dem Beginn einer neuen Entwicklung: Berechnet man den Bedarf an Pflegeplätzen ausschließlich auf der Grundlage der prognostizierten Bevölkerungsentwicklung und schreibt man die gegenwärtigen Pflegebedürftigkeitsquoten in die Zukunft fort, so ge- 
langt man zu dem Ergebnis, dass jährlich 200 bis 250 stationäre Pflegeeinrichtungen neu errichtet werden müssen. Wird zudem noch berücksichtigt, dass die derzeit betriebenen Pflegeeinrichtungen häufig nicht mehr den aktuellen medizinischen und pflegewissenschaftlichen Anforderungen entsprechen, so ergibt sich ein zusätzlicher Ersatzbedarf.

Diese Aussichten sind Grundlage einer Goldgräberstimmung, die momentan die Diskussion beherrscht. Begünstigt wird diese Entwicklung durch die Umstellung der staatlichen Unterstützung von der Objekt- auf die Subjektförderung, die bewusst privates Kapital und Wettbewerb in den Markt ziehen will. Wichtig ist aber, dass ein privater Investor die Kriterien kennt, die die Vorteilhaftigkeit seiner Investition in diesem Immobilienbereich bestimmen.

\section{Renditekriterium Standort}

Einige private Investitionen in Pflegeimmobilien sind in der Vergangenheit an einem falsch ausgewählten Standort gescheitert. Entscheidend ist einerseits die gegenwärtige und zukünftige Bevölkerungsstruktur eines möglichen Standortes. In diesem Zusammenhang sind die Alterszusammensetzung und deren Prognose - unter Berücksichtigung möglicher Wanderungsbewegungen für die kommenden Jahrzehnte zugrunde zu legen. Aufgrund derzeit bereits vorliegender wissenschaftlicher Studien (z. B. der Bertelsmann Stiftung) lässt sich die zukünftige Bevölkerungsentwicklung gut vorhersagen.

Darüber hinaus bedarf es einer sorgfältigen Analyse des Wettbewerbumfelds. Hierzu müssen die bestehenden stationären Pflegekapazitäten und deren Auslastung an dem möglichen Standort sowie die derzeit geplanten Investitionen untersucht werden. Besonders bedeutsam ist in diesem Zusammenhang auch die Frage, ob sich die bestehenden und geplanten Pflegeeinrichtungen auf bestimmte Bereiche (z. B. gerontopsychiatrische Krankheitsbilder) spezialisiert haben. Die Standortentscheidung wird zudem durch die Kaufkraft der Bevölkerung und das Angebot an Arbeitskräften im Bereich der Pflegeberufe beeinflusst.

Grundsätzlich geht die Tendenz zu einer eher stadtteilnahen Pflege, sodass Pflegeeinrichtungen auf der grünen Wiese wenig attraktiv erscheinen, weil höhere Leerstandsquoten zu befürchten sind. Der große Bedarf an zukünftigen Pflegekapazitäten wird sich gesellschaftspolitisch nur realisieren lassen, wenn die Pflege und Betreuung der älteren Menschen in ihrem bisherigen Wohnquartier erfolgt, um die Eigenständigkeit der Bewohner möglichst lange zu erhalten. Allerdings ist zu berücksichtigen, dass für spezielle Krankheitsbilder (z. B. Demenz im fortgeschrittenen Stadium, Wachkomapatienten) ein belebtes Umfeld für die Bewohner ohne Bedeutung ist. Derartige Pflegeeinrichtungen können auch in städtischen Randlagen gegründet werden, sodass aufgrund der geringen Grundstückspreise größere Flächen realisiert werden können. Dies wiederum fördert bestimmte Pflegekonzepte, da beispielsweise bei der Demenzbetreuung ein großzügiges Platzangebot für (geschlossene) Außenflächen vorgehalten werden sollte.

\section{Renditekriterium Betreiber}

Pflegeimmobilien gehören zu den klassischen »Betreiberimmobilien «, die wirtschaftliche Leistungsfähigkeit des (einzigen) Betreibers sichert also die Miete, die der Investor für die Überlassung der Immobilie erhält. Ein Austausch des Betreibers ist oft nicht oder nur mit hohem Aufwand möglich. Betreiberimmobilien werden oftmals speziell nach den Bedürfnissen eines bereits vor Errichtung gefundenen Betreibers konzipiert, sodass eine Drittverwertungsmöglichkeit nach Ende des (normalerweise langfristigen) Mietvertrages oder eine Überlassung an einen anderen Betreiber nur eingeschränkt möglich ist. Betreiber und Investor sind in dieser Konstellation in besonderem Maße aneinander gebunden.

Vor diesem Hintergrund ist eine sorgfältige Analyse des Betreibers und - bei positivem Urteil - eine ernsthafte Kooperationsvereinbarung unerlässlich. Dabei sind weniger klassische betriebswirtschaftliche Kennzahlen (z. B. die Eigenkapitalquote) als vielmehr die bisherige Erfahrung des Betreibers und insbesondere seine strategische Ausrichtung von Bedeutung. Gerade der letzte Punkt schränkt die möglichen Kooperationspartner für einen privaten Investor ein. Der Betreiber muss in der Lage und bereit sein, seine Marktposition aufgrund innovativer Pflege- und Betreuungskonzepte auszubauen. Eine Konzentration auf bestimmte Krankheitsbilder (z. B. gerontopsychiatrische Krankheiten) bietet sich an, da eine qualitativ hochwertige Dienstleistung auch in diesem Bereich eine Spezialisierung erfordert.

Zudem ist von Bedeutung, ob der Betreiber bereit ist, mit einem Privatinvestor zu kooperieren, der eigene betriebswirtschaftliche Interessen verfolgt. In der Praxis ist $\mathrm{zu}$ beobachten, dass insbesondere öffentliche und freigemeinnützige Betreiber teilweise Schwierigkeit haben, beispielsweise einen indexierten Mietvertrag zu akzeptieren, der eine automatische Mietzinserhöhung in Abhängigkeit von der Steigerung eines Lebenshaltungsindex vorsieht. Aufgrund der Langfristigkeit der Bindung und der unsicheren Zukunft der Einnahmenseite des Betreibers (Investitionskostenanteil im Pflegesatz) sind viele Betreiber in diesem Punkt eher zurückhaltend.

Ein privater Investor wird zudem versuchen, das Betreiberrisiko dadurch zu vermindern, dass er seine Informationsmöglichkeiten über den laufenden Betrieb der Pflegeeinrichtung ausweitet. Dies betrifft einerseits die Daten über die wirtschaftliche Lage des Betreibers (zeitnahe Vorlage von Jahresabschlüssen und Zwischenberichten), andererseits aber auch Unterlagen zur Etablierung und Auswertung von sinnvollen Qualitätssicherungssystemen. Diese Informationen sichern letztlich das Kapital des Investors und sollten daher vom Betreiber nicht als Belastung, sondern als Chance zur Transparenz verstanden werden.

\section{Renditekriterium Pflegeversicherung}

Seit der Einführung der Pflegeversicherung ist die Zahl der stationären Pflegeeinrichtungen stetig gestiegen. In den vergangenen Jahren hat kein Segment so starke 
Wachstumsraten gehabt wie die Zahl der Bewohner der stationären Pflegeeinrichtungen mit der Pflegestufe I. Diese Entwicklung wird oftmals als Beweis des weiter andauernden Bedarfes nach stationären Pflegeeinrichtungen auch für leicht pflegebedürftige Bewohner - herangezogen. Fraglich ist aber, ob sich in der Vergangenheit nicht das Angebot die Nachfrage selbst geschaffen hat. Teilweise wurde eine Unterbelegung durch die Aufnahme von Bewohnern kompensiert, deren gesundheitlicher Zustand nicht unbedingt einen Heimaufenthalt rechtfertigt. Auch die Pflegekassen prüfen nach einer zugeteilten Pflegestufe regelmäßig nicht die Notwendigkeit einer Unterbringung in einer stationären Pflegeeinrichtung.

Wenn auch generell von einem weiter steigenden Bedarf an stationären Pflegeeinrichtungen auszugehen ist, so scheint die einfache lineare Fortschreibung der gegenwärtigen Verhältnisse zu falschen Erwartungen zu führen. Es besteht weitgehend Einigkeit darüber, dass die Pflegeversicherung den rechnerischen Anstieg der stationären Pflegeplätze nicht finanzieren kann. Um den endgültigen Zusammenbruch der Pflegekassen zu vermeiden, wird bereits jetzt ein Maßnahmenbündel diskutiert, das von einer Absenkung der Pflegeleistungen in der Pflegestufe I auf das Niveau der ambulanten Pflege bis zu einer Umstellung der Pflegeversicherung auf ein kapitalgedecktes System reicht.

Wenn die Bewohner der stationären Pflegeeinrichtungen nur noch reduzierte Leistungen aus der Pflegeversicherung erhalten, werden - sofern nicht ausreichend eigenes Einkommen und Vermögen vorhanden ist - die Sozialhilfeträger die anfallenden Kosten übernehmen müssen. Diese wiederum werden versuchen, möglichst kostengünstige Einrichtungen zu belegen. So wird beispielsweise die heute vorherrschende Unterbringung in Einzelzimmern zukünftig eher die Ausnahme sein. Im Rahmen der baulichen Konzeption der heute neu zu errichtenden Pflegeeinrichtungen ist diese wahrscheinliche Entwicklung bereits durch eine flexible Grundrissplanung $\mathrm{zu}$ berücksichtigen.

\section{Resümee}

Wirtschaftlich erfolgreiche Betreiberkonzepte müssen sich den bestehenden Finanzierungsunsicherheiten stellen. Zukunftsweisend sind daher keine isolierten Pflegeheime, sondern »Pflegezentren «, die neben einem stationären Bereich auch neue Wohnformen (z. B. ambulant betreute Wohngruppen) umfassen. Durch die Integration von Tages- oder Kurzzeitpflege (z. B. nach einem längeren Krankenhausaufenthalt) können die Betreiber die komplette Wertschöpfungskette abbilden und flexibel auf Veränderungen reagieren.

Die bisher vorherrschende Trennung zwischen dem stationären und dem ambulanten Bereich muss - wie auch in anderen Bereichen des Gesundheitswesens - durch eine verstärkte Kooperation des Betreibers beispielsweise mit Krankenhäusern überwunden werden. Ein privater Investor wird daher auch darauf achten müssen, ob sein Betreiber diese Anforderungen erfüllen kann oder will.

\section{„Investitionen in Pflegeimmobilien lohnen - wenn die verän- derten Rahmenbedingungen berücksichtigt werden«}

Als »unsolide und irreführend« haben Vertreter führender Unternehmen der Altenpflege Schätzungen bezeichnet, nach denen bis 2020 rund 300.000 neue stationäre Pflegeplätze in Deutschland benötigt werden. Unterstellt man Investitionen von zirka 100.000 Euro pro Pflegeplatz, würde dies einem Investitionsvolumen von bis zu 30 Milliarden Euro entsprechen. Zwischen 2.500 bis 5.000 neue Pflegeheime müssten gebaut werden, so die Prognosen. Die in der »Zukunftswerkstatt« zusammenarbeitenden Fachleute warnten vor überhöhten Renditeerwartungen auf dem Markt der Pflegeimmobilien. In der »Zukunftswerkstatt» haben sich im Jahr 2001 Anbieter von Dienstleistungen für Senioren zusammengeschlossen.

Basis der Studien ist der demografische Wandel und der daraus resultierende wachsende Anteil älterer und hochbetagter Menschen. Daraus schließen Banken und Marktforscher, dadurch steige zwangsläufig der Bedarf an stationären Pflegeplätzen. Diese Annahme sei falsch und fachlich durch nichts begründet, so Ingrid Hastedt, Vorstandsvorsitzende des Wohlfahrtswerkes für Baden Württemberg und eine der Sprecherinnen der »Zukunftswerkstatt«. Unterstützt wird diese Ansicht von Dr. Helmut Braun, Vorstandsvorsitzender Kuratorium Wohnen im Alter gAG, Dr. Udo Krolzik, Vorsitzender des Vorstands des Ev. Johanneswerks e. V., Alexander Künzel, Vorstandsvorsitzender der Bremer Heimstiftung, Franz I. Stoffer, Ceschäftsführer der Caritas-Betriebsführungs- und Trägergesellschaft mbH und Helmut Wallrafen-Dreisow, Geschäftsführer der Sozial-Holding der Stadt Mönchengladbach $\mathrm{CmbH}$.

Die derzeitige Bedarfsdiskussion werde aus seiner Sicht nur immobilienorientiert, aber nicht fachlich begründet geführt, betont Dr. Braun. Die vorliegenden Studien übersähen, dass Pflege nach wie vor allem von Familien und privaten Netzwerken geleistet werde. Zudem verzeichneten das größte Wachstum derzeit alternative Wohn- und Pflegekonzepte sowie Dienstleistungsangebote, die eine Pflege zu Hause ermöglichen - nicht im stationären Bereich.

Im Cegensatz zu einer Reihe von Finanz- und Marktforschungsinstituten gehen die Vertreter der »Zukunftswerkstatt« zwar ebenfalls von einem Marktwachstum aus. Dieses wird jedoch ihrer Ansicht nach weitgehend von einer deutlichen Erweiterung der Angebotspalette aufgefangen. Es werde sicherlich mehr Pflegebedürttige geben, aber eben auch mehr und unterschiedliche Angebote - von einer Ausweitung ambulanter Betreuung bis hin zu WG-, Wohngruppen- und anderen neuen Wohnkonzepten. Statt stationärer Pflege von der Stange erwarteten ältere Menschen heute individuelle Konzepte, die innen erlaubten, die eigene Unabhängigkeit zu erhalten, so Ingrid Hastedt.

Die Prognose der »Zukunftswerkstatt» lautet daher: Der Markt der Pflege wird sich nicht einseitig im stationären Bereich entwickeln, sondern ein Bündel von Versorgungspfaden anbieten, die den vielfältigen Interessen der betroffenen Menschen entsprechen. Der künftige Bedarf wird bestimmt durch regionale Konzepte, vernetzte Strukturen, neue Wohnformen, also eine stärkere Differenzierung des Angebotes.

Ein weiterer Aspekt, der das stetige Wachstum stationärer Pflegeeinrichtungen fragwürdig erscheinen lässt, ist der Kostenfaktor: Bereits heute werden die Leistungen der Pflegekassenleistungen oft eher als Einkommensbestandteil der Haushalte der pflegenden Angehörigen verwendet als tatsächlich zur Abdeckung von Pflegekosten benutzt. Zudem wird die geplante Cleichstellung ambulanter und stationärer Pflege durch die Pflegeversicherung zu einer Verschiebung zugunsten ambulanter Leistungen führen. Der Kostenvorteil, den die stationäre Unterbringung eines Pflegebedürttigen noch heute bringe, werde entfallen, so Dr. Braun. In diesem Zusammenhang sind auch Restriktionen der Sozialhilfeträger in Bezug auf stationäre Versorgung zu erwarten, sobald die Pflegekassenleistungen Pflegeheimbewohner nicht mehr gegenüber ambulant Versorgten bevorzugen. Dies wird aus Sicht der »Zukunftswerkstatt» dazu führen, dass der prognostizierte stationäre Pflegeplatzbedarf bei weitem nicht erreicht werden wird. Derzeitige Renditevorstellungen der Investoren sind dann nachhaltig nicht zu erzielen.

Lohnen sich also Investitionen in Pflegeimmobilien nicht? Doch, sagt die »Zukunftswerkstatt«, so weit die veränderten Rahmenbedingungen berücksichtigt werden. Die Experten raten Anlegern daher, in Konzepte zu investieren, die:

- Dienstleistungen und die Erhaltung der Individualität in den Mittelpunkt stellen

- den Fokus auf Pflege- und Betreuungsqualität legen

- nicht einseitig die Erhöhung der Zahl stationärer Plätze vorsehen

- die Vielfalt von Betreuungs- und Dienstleistungsangeboten fördern.

Kontakt: Business Network, Fasanenstraße 47, 10719 Berlin, Telefon 030 81464600, Fax 030 814646046, E- Mail sk@businessnetwork-berlin.com 